\title{
Effects of Heavy Gases on the Tandem Mass Spectra of Peptide Ions in the Quadrupole Ion Trap
}

\author{
Richard W. Vachet and Gary L. Glish \\ Department of Chemistry, University of North Carolina, Chapel Hill, North Carolina, USA
}

Heavy gases (xenon, argon, krypton, methane) have been used to improve the performance
of the quadrupole ion trap when performing collision-induced dissociation on peptides.
MS/MS spectra reveal that increased amounts of internal energy can be deposited into
peptide ions and more structural information can be obtained. Specifically, the pulsed
introduction of the heavy gases (as reported previously by Doroshenko, V. M.; Cotter, R. J.
Anal. Chem. 1996, 68,463 ) provides greater energy deposition without the deleterious effects
that static pressures of heavy gas have on spectra. Internal energy deposition as indicated by
a qualitative evaluation of MS /MS spectra shows pulsed introduction of heavy gases enables
ions to obtain more internal energy than possible by using static pressures of the same heavy
gases. A linear correlation is observed between the percentage of heavy gas added and the
ratio of product ions used to reflect internal energy deposition. Results here also show that
upon pulsed introduction of heavy gases, empirical optimization of a single frequency
resonant excitation signal is no longer needed to obtain good MS /MS spectrometry effi-
ciency. The presence of many low mass-to-charge ratio ions and the absence of side chain
cleavages in the MS/MS spectra of peptides suggests that the propensity for consecutive
fragmentations is increased with the pulsed introduction of heavy gases. In addition, by
varying the delay time between introduction of the gas and application of the resonant
excitation signal, the amount of fragmentation observed in MS/MS spectra can be changed.
(c) 1996 American Society for Mass Spectrometry (J Am Soc Mass Spectrom 1996, 7, 1194-1202)

$\mathrm{I}$ $n$ recent years the quadrupole ion trap has developed into a powerful mass spectrometer [1, 2]. An important part of the development of the quadrupole ion trap has been various capabilities to manipulate and control ions to perform tandem mass spectrometry (MS/MS) experiments. Many of these different types of MS/MS experiments can be effected with high efficiency, providing sensitive structural analysis. The most common of the MS/MS techniques is collision-induced dissociation (CID), and often selected parent ions can be converted to product ions with MS/MS efficiencies of $75-100 \%$ [3].

Although CID in the quadrupole ion trap has proven to be very useful, there are some limitations to the traditional manner it is performed. Among these is the fact that the amount of internal energy that is typically imparted into an ion during resonant excitation is relatively small [2]. This is due primarily to the low center-of-mass collision energies involved during CID in the presence of helium. Also, the mass-to-charge ratio range over which product ions can be efficiently

\footnotetext{
Address reprint requests to Dr. Gary L. Glish, Department of Chemistry, CB 3290, University of North Carolina, Chapel Hill, NC 275993290.
}

observed is limited. The low mass cutoff depends on the fundamental rf voltage $\left(q_{z}\right)$ and may prevent the trapping of low mass product ions formed during CID. To observe these low mass product ions, the parent ion should be collisionally activated at the lowest possible $q_{z}$ value. Experimentally, though, there is a compromise between performing CID at low $q_{z}$ values and efficiently producing fragment ions because as $q_{z}$ is lowered resonant ejection becomes competitive with CID [4].

Another limitation in traditional CID is that the application of a resonance excitation signal to the endcaps at the theoretically predicted frequency for ions of a given mass-to-charge ratio rarely results in efficient (or any) dissociation of that ion. Space-charge effects and higher order fields (present due to imperfections in the geometry of the quadrupole ion trap) cause the secular frequencies of ions to be shifted from that theoretically predicted [5-8]. The optimum experimental resonant frequency in most cases must be empirically determined and this process can be time consuming. Furthermore, this optimum resonant frequency shifts as the excitation voltage is changed [9].

In an attempt to overcome some of these limitations of CID in the quadrupole ion trap, a number of approaches have been proposed. Experiments have been 
described in which mass selected ions undergo collisions with the ring electrode via the application of a rapid dc pulse to the endcaps [10]. These experiments result in greater energy deposition but at the cost of MS/MS efficiency. Another excitation method that does not require applying a resonant signal is referred to as "boundary" activation [11-15]. This method, however, also results in low efficiencies and conflicting results have been reported with regard to energy deposition $[13,14]$. The use of heavy gases (argon, xenon, etc.) to increase the energy deposition has been demonstrated [4, 16-18]. In fact Doroshenko and Cotter [19] recently described experiments in which heavy gases are pulsed in just prior to the MS/MS stage of an experiment. They demonstrated that this mode of operation allows CID to be performed with good efficiency at $q_{z}$ values as low as 0.05 when applying a broadband excitation signal via a stored waveform (SWIFT). They also presented results that show low mass-to-charge ratio $(\mathrm{m} / \mathrm{z})$ product ions of peptides not normally observed in quadrupole ion trap CID spectra are observed with the pulsed introduction of heavy gases. Our laboratory concurrently has been performing experiments in which heavy gases are introduced by a pulsed valve just prior to the MS/MS stage of operation, and our results agree well with Doroshenko and Cotter's data.

We present here a more detailed study of the effects of pulsed introduction of heavy gases on CID when performed by single frequency resonant excitation. Specifically, the effects on internal energy deposition, the frequency of the excitation signal, and a means of varying the MS/MS spectra will be described. Our results demonstrate that the limitations of low energy deposition and the empirical optimization of the resonant excitation frequency can be overcome by pulsed introduction of heavy gases. Additionally, we present explanations for the loss of resolution and sensitivity when heavy gases are present at static pressures throughout the complete experimental sequence and the reasons for the potential appearance of apparent peaks at both lower and higher masses.

\section{Experimental}

Experiments were performed on a Finnigan MAT ITMS $^{\mathrm{TM}}$ (San Jose, CA) modified with a custom-built electrospray source of similar design to one previously reported [20]. A model 22 Harvard Apparatus (South Natick, MA) syringe pump was used to infuse solutions at $0.25-0.50 \mu \mathrm{L} / \mathrm{min}$ into a capillary that was connected to a needle via a Teflon union. The needle voltage was held at $4-4.5 \mathrm{kV}$.

Krypton, xenon, argon, methane, and helium were introduced either by a variable leak valve or by a series 9 high speed solenoid valve, with an exit orifice of $1.0 \mathrm{~mm}$, that was mounted on the vacuum manifold. Small ceramic spacers separated the ring electrode from each endcap electrode which allowed free conduction of gases into the volume of electrodes. The solenoid valve was actuated by means of a General Valve Corporation (Fairfield, NJ) Iota One pulse driver that supplied a TTL pulse of variable length. The pulse driver was triggered by another TTL pulse from the ITMS electronics. Solenoid pulse lengths generally ranged between 250 and $2000 \mu$ s. Heavy gas inlet pressures ranged from 200 to 600 torr. A static helium pressure of $8.6 \times 10^{-4}$ torr (corrected) was used in all cases. In all experiments a $2.8 \times 10^{-5}$ torr pressure of air was present. The air pressure corresponded to a partial pressure of about $3 \%$.

Figure 1 illustrates a typical scan function employed in these studies. Electrospray generated ions were injected into the ion trap through an endcap for a period of 200-400 ms. The selected parent ion mass-to-charge ratio was then isolated by ramping the rf voltage to a level that ejected all the ions below the ion of interest. For parent ions of mass-to-charge ratio greater than $650 \mathrm{u}$ a supplementary ac signal was applied to the endcaps during the $\mathrm{rf}$ ramp to resonantly eject all ions with mass-to-charge ratios above 650 and below the parent ion of interest. The rf voltage was then ramped back down to a level that allowed ions of the mass-tocharge ratio of interest to be stored at the desired $q_{z}$ value. Next, the ITMS electronics sent a pulse to trigger the Iota One pulse driver and the heavy gas was pulsed into the vacuum system. A variable delay (10-150 ms) was added to allow time for the gas to diffuse into the volume of the electrodes. This variable delay also permitted the number density of the gas, during the CID portion of the scan function, to be changed. The ions then were resonantly excited by the application of a 20 -ms supplementary ac signal to the endcaps. Another delay time $(200-400 \mathrm{~ms})$ was then added to ensure the evacuation of the heavy gas from the vacuum system before acquisition of the spectrum. In all cases (except those noted) the resonant excitation frequency during CID was empirically optimized from the theoretically predicted frequency to provide maximum dissociation of the selected parent ion.

The peptides, des-Arg ${ }^{9}$-bradykinin (RPPGFSPF) and leucine enkephalin (YGGFL), were obtained from

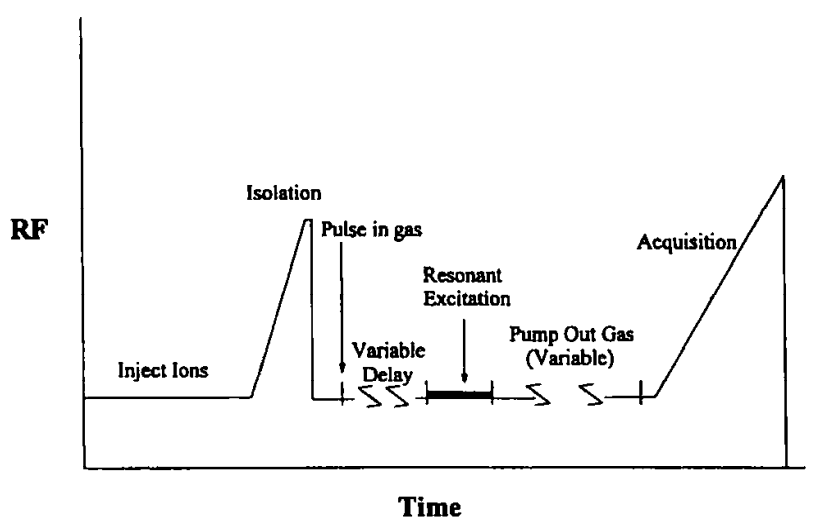

Figure 1. Typical scan function for performing MS/MS while pulsing in heavy gases. 
Sigma Chemical Company (St. Louis, MO). These peptides were dissolved in a 20:75:5 mixture of water: methanol: acetic acid to a concentration of $250 \mu \mathrm{M}$. MS/MS data were obtained on singly protonated species and from 100-300 scans were averaged to give a spectrum. The large number of scans in some cases were used to provide good signal-to-noise for low intensity product ion signals that were often present in MS/MS spectra in which ion intensity was spread out over as many as 45 product ions.

Ion motion simulations were performed by using ITSIM [21], which was obtained from the Cooks group at Purdue University. This simulation program allowed ion trap operating parameters (rf voltage, background gas pressure, etc.) to be varied and the resulting ion motion and kinetic energy to be calculated. During the simulations the rf voltage was set to store the ion at the desired $q_{z}$ value. The ions were allowed to cool for $20 \mathrm{~ms}$, after which supplementary ac signals at varying amplitudes were applied to resonantly excite ions for $10 \mathrm{~ms}$. During this time the resonantly excited ion reached a maximum kinetic energy which stayed constant. When the resonant excitation signal was removed, the time for the kinetic energy to be dampened to $0.01 \mathrm{eV}$ was determined.

\section{Results and Discussion}

It has been previously reported that a small percentage of heavy gas, such as xenon, present with the normal helium bath gas enables the dissociation of ions that are typically difficult to fragment via CID in the quadrupole ion trap [17]. Also, signals from product ions of dissociation pathways normally not observed have been enhanced by the presence of a small percentage of a heavy gas [17]. To study the effects of static pressures of heavier gases on the dissociation behavior of peptide ions, leucine enkephalin was chosen as a model system. Alexander and Boyd [22] noted that the ratio of intensities of the $b_{3}$ ion from protonated leucine enkephalin to the $\mathbf{y}_{2}$ ion serves as a good indication of the amount of energy deposited into the parent ion. In later studies, however, they suggested that perhaps a better gauge of the internal energy deposition is the ratio of the intensities of the $a_{4}$ ion to the $b_{4}$ ion [23]. Due to the greater abundances of the $\mathbf{a}_{4}$ and $\mathbf{b}_{4}$ ions in the quadrupole ion trap CID spectra, their intensities were chosen as the gauge of internal energy deposition. Figure 2 shows a plot of the $\mathbf{a}_{4} / \mathbf{b}_{4}$ intensity ratio with respect to the percentage of heavy gas added for various gases. The plots show that there is a fairly good linear correlation between the amount of gas added and ratio of the two peaks. As was expected and noted before [16], increasing pressures of heavy gases result in greater energy deposition.

This linear relationship is what one might expect. As the percentage of heavy gas is increased, the amount of internal energy that can be deposited will increase due to increased number of collisions with the heavy

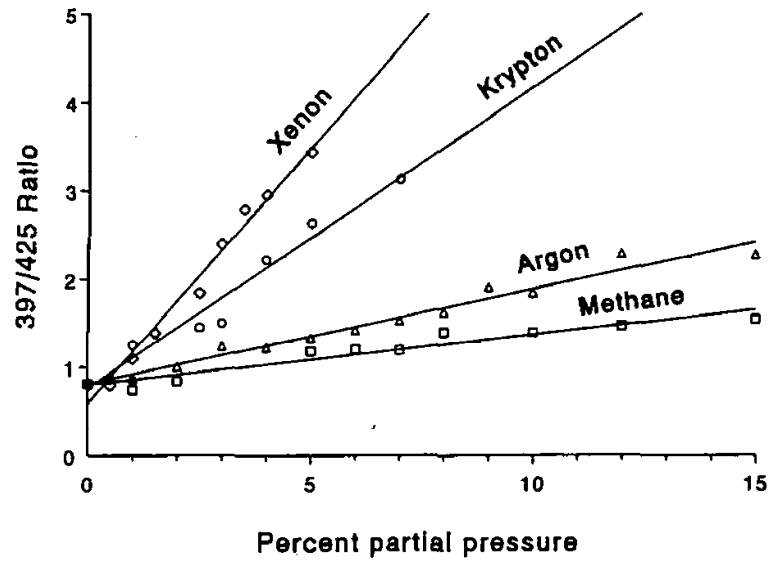

Figure 2. Plot of the ratio of the intensity of the $\mathbf{a}_{4}$ ion to the $\mathbf{b}_{4}$ ion of leucine enkephalin with respect to percent partial pressure of heavy gases in a mixture with helium $(O$, xenon; $O$, krypton; $\Delta$, argon; $\square$, methane).

gas. The increased number of collisions with the heavy gas gives an overall increase in internal energy despite the reduction in the number of collisions with helium. A plot of internal energy (calculated by summing the center-of-mass collision energies for collisions with helium and a given heavy gas, weighted by the percentage of collisions with helium and the heavy gas, respectively) versus percentage of heavy gas results in a straight line.

The graph (Figure 2) also shows that slopes of the lines for the various gases increase with the increasing mass of the added gas. The trend of increasing slopes is what one might expect if the center-of-mass collision energy $\left(E_{\text {com }}\right)$ is considered:

$$
E_{\mathrm{com}}=E_{\mathrm{lab}}\left(\frac{M_{\mathrm{n}}}{M_{\mathrm{p}}+M_{\mathrm{n}}}\right),
$$

where $E_{\text {lab }}$ is the ion's laboratory frame kinetic energy, $M_{n}$ is the mass of the neutral target, and $M_{p}$ is the mass of the ion. As the mass of the neutral target is increased, $E_{\mathrm{com}}$ is also increased, which enables more energy to be converted from kinetic to internal. However, it might be expected that the slopes of the lines for the different gases would increase proportionately with the increase in $E_{\text {com }}$, but this is not the case. Table 1 shows the center-of-mass collision energy and the slopes from Figure 2 for the four gases (xenon, krypton, argon, and methane). The center-of-mass collision

Table 1. Polarizabilities, slopes from Figure 2, normalized slopes, and normalized center-of-mass collision energies for collisions of various gases with leucine enkephalin

\begin{tabular}{lcccc}
\hline Heavy gas & Polarizability & $\mathrm{E}_{\text {com }}^{*}$ & Slope & Slope $^{*}$ \\
\hline \hline Xenon & 4.044 & 6.82 & 0.579 & 10.2 \\
Krypton & 2.484 & 4.68 & 0.341 & 5.98 \\
Argon & 1.641 & 2.39 & 0.108 & 1.89 \\
Methane & 2.593 & 1.00 & 0.057 & 1.00 \\
\hline
\end{tabular}


energies have been normalized to the value of methane $\left(E_{\mathrm{com}}^{*}\right) . E_{\mathrm{com}}^{*}$ is calculated based on eq 1 and assuming that the lab-frame kinetic energies of the ions are the same in the collisions with the different gases. The slopes of the lines also have been normalized (slope*) to the slope obtained from the line involving CID with methane. This normalization was done to make the comparison between $E_{\mathrm{com}}$ and the slopes of the lines easier to see. As can be observed readily, the slope* values are not directly proportional with the $E_{\mathrm{com}}^{*}$ values. In the case of xenon, the normalized slope is much greater than $E_{\text {com }}^{*}$. This most likely can be attributed to xenon's greater polarizability (also listed in Table 1) with respect to methane. The most efficient form of kinetic to internal energy conversion is by the formation of a long-lived collision complex, and the formation of a collision complex is maximized when a polarizable target is used [24]. Therefore, the higher than expected slope in the case of xenon is mostly attributed to its greater propensity to form longer-lived collision complexes and efficiently convert kinetic energy into internal energy. The slightly higher than expected slope in the case of krypton, relative to methane, requires a different explanation because krypton's polarizability is very similar to that of methane's. Due to the fact that methane is polyatomic, whereas krypton is monoatomic, a fraction of $E_{\mathrm{com}}$ in the collision between leucine enkephalin and methane can be transferred into vibrational modes of methane. Xenon and argon are also monoatomic, but their polarizability with respect to methane is the dominant difference that explains the slopes observed when these heavy gases are used. Argon has a lower polarizability than methane, so it is probable that a collision with argon results in a smaller percentage of $E_{\text {com }}$ being converted into the internal energy of leucine enkephalin, when compared to a collision with methane. This would explain the lower than expected slope, based on $E_{\text {com }}$ values, in the case of CID with argon.

In Figure 2 it is clear that xenon is the most efficient at depositing internal energy. The addition of up to $5 \%$ xenon considerably increases the amount of fragmentation observed in the MS/MS spectra. Figure 3 shows the MS/MS spectra of leucine enkephalin at $q_{z}=0.229$ and resonant excitation voltage of $325 \mathrm{mV}$ with (a) no xenon and (b) $5 \%$ xenon. The addition of $5 \%$ xenon to the background helium results in a MS/MS spectrum that has greater intensities of lower mass-to-charge ratio product ions than the MS/MS spectrum in the presence of helium alone.

While increasing the static pressure of the heavy gases leads to higher energy deposition, this is at the expense of resolution and sensitivity. Figure 4 shows the mass spectra in the region of the singly protonated species of leucine enkephalin at various percentages of xenon. It is clear that a normal mass spectrum is obtained in the absence of xenon (Figure 4a). With the addition of $5 \%$ xenon (Figure $4 \mathrm{~b}$ ), the isotropic distri-
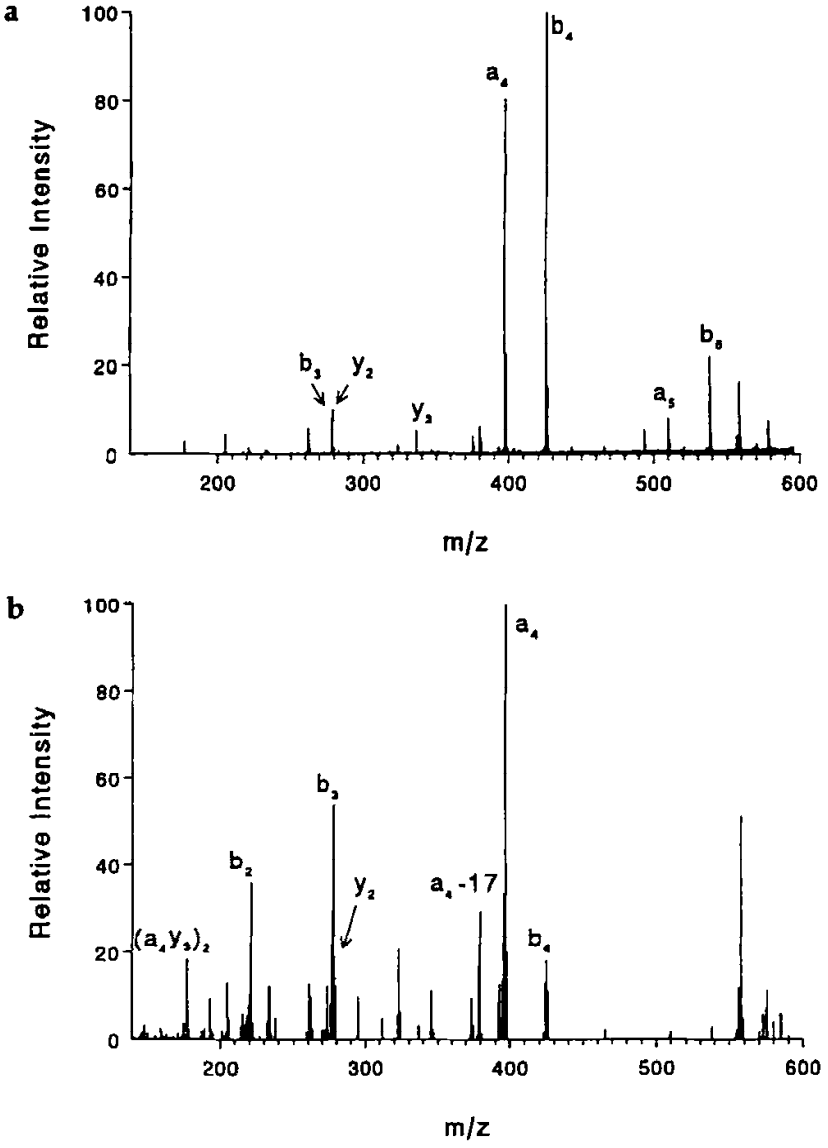

Figure 3. (a) MS/MS of leucine enkephalin in the presence of helium only at $q_{z}=0.229$. (b) MS/MS of leucine enkephalin with $5 \%$ partial pressure of xenon at $q_{z}=0.229$.

bution becomes distorted. Also, the intensity of the protonated species is reduced as the pressure of xenon is increased. Furthermore, an apparent reduction in resolution can be observed in Figure 4. The loss in sensitivity and resolution can be attributed to the scattering and dissociation of the ions just prior to ejection and detection. During the $\mathrm{rf}$ acquisition scan, resonant ejection is performed by the application of a supplementary ac signal to the endcaps at a frequency of 530 $\mathrm{kHz}$. As the protonated molecule's $q_{z}$ value is increased to approach a value of 0.908 , the ion's motion in the axial direction increases in response to the applied supplementary signal. As the ion's motion is increased in the axial direction it can undergo energetic collisions with xenon that lead to dissociation in which the subsequent product ion that is formed has a $q_{z}$ value greater than 0.908 . This product ion is immediately ejected, resulting in ion current at the electron multiplier corresponding to mass-to-charge ratio values slightly lower than the protonated species. This apparent shift to lower mass-to-charge ratio values can be clearly seen in the spectrum in Figure $4 \mathrm{~b}$. The reduction of the signal and the subsequent loss of sensitivity can be explained by the scattering of ions from their trajectory to the detector by collisions with xenon. 

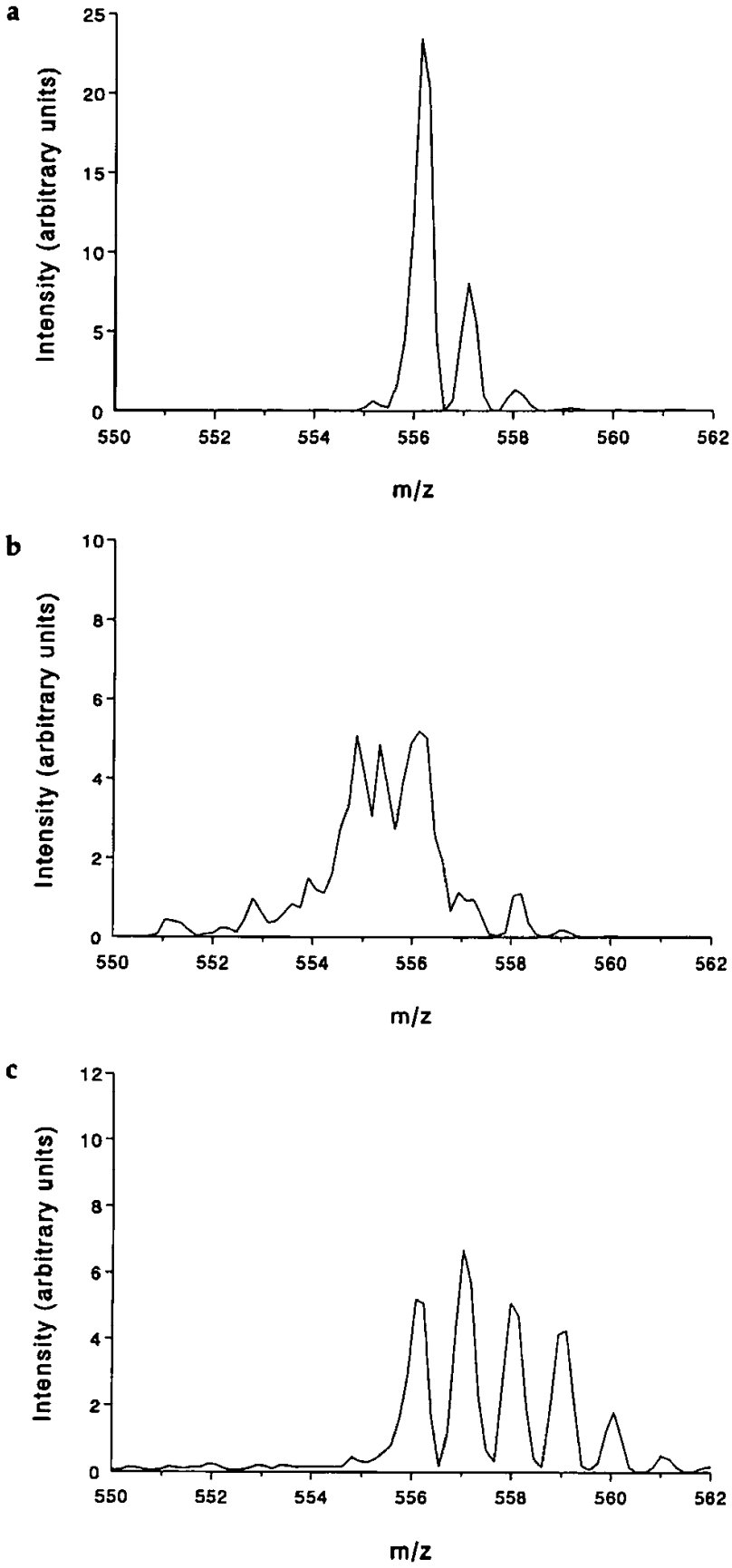

Figure 4. Mass spectra of leucine enkephalin in the region of the protonated molecule in the presence of helium alone (a) and $5 \%$ xenon (b). Mass spectrum of leucine enkephalin in the region of the protonated molecule acquired during resonant ejection at $q_{z}=0.901$ in the presence of $2 \%$ xenon (c).

Static pressures of heavy gases will also affect the performance of resonant ejection when extending the mass-to-charge ratio range. Figure $4 \mathrm{c}$ shows the mass spectrum obtained for leucine enkephalin when the resonant ejection signal is applied at a frequency of 510 $\mathrm{kHz}$ to slightly extend the mass range. In this figure the mass-to-charge ratio values have been corrected by considering the $q_{z}$ value at which the ions are resonantly ejected $\left[q_{z}\right.$ (eject) $\left.=0.901\right]$. The spectrum does not observably change in the presence of helium alone (i.e., it is the same as Figure 4a). However, in the presence of $2 \%$ xenon (Figure $4 \mathrm{c}$ ), multiple peaks are observed including ones "shifted" to higher mass-tocharge ratio. These results can be explained by considering that resonant ejection at $q_{z}$ values below 0.908 involves increasing the motion of ions by the application of a supplementary signal so that ion trajectories are no longer stable, and the ions are ejected to the detector at a point other than the normal boundary at $q_{z}=0.908$. During resonant ejection in the presence of xenon, ion motion can be effectively damped to prevent some ions from being ejected and detected. With continued scanning of the rf amplitude, the ions that were not ejected are no longer in resonance, and the amplitude of their motion is reduced until the stability edge at $q_{z}=0.908$ is approached. At the stability edge, the remaining ions will then be ejected via mass selective instability and give rise to a signal on the detector at higher apparent mass-to-charge ratio values. This incomplete ejection of ions has implications when extending the mass-to-charge ratio range by resonant ejection in the presence of heavy gases. Thus to ensure that all the ions are ejected when they come into resonance with the applied resonant ejection signal, the amplitude of signal has to be increased when heavy gases are present.

A means of circumventing the loss in resolution and sensitivity, while continuing to take advantage of the increased energy deposition afforded by the presence of the heavy gases during CID, is to have the heavy gases present only during the CID stage of the experiment. This can be achieved by the pulsed introduction of the heavy gas immediately prior to resonant excitation. When accompanied by sufficient time to allow the gas to be pumped away before acquisition of the spectrum, the deleterious effects on resolution and sensitivity can be overcome while maintaining the desired increase in energy deposition during CID. The mass spectrum in the region of the protonated species of leucine enkephalin yields a result nearly identical to Figure $4 \mathrm{a}$ when xenon is pulsed in and then allowed to be pumped away for $300 \mathrm{~ms}$ before data acquisition.

Another benefit of the pulsed introduction of the heavy gases is the increased number density of gas atoms that can be present during CID. The increased number density allows a greater amount of energy to be deposited into ions when compared to a static pressure of gas. For example, the MS/MS spectrum of leucine enkephalin after a $500-\mu \mathrm{s}$ pulse of krypton results in an $\mathbf{a}_{4}$ to $\boldsymbol{b}_{4}$ ratio of 7.5 . From Figure 2, assuming the linear relationship remains at higher pressures, to achieve a similar result with a static pressure of krypton, the heavy gas would have to be present at $24 \%$ of the total gas pressure. With a static pressure of krypton at this level, no ion signal can be observed.

Figure 5 shows an example of how the increased energy deposition can facilitate obtaining structural information for larger peptides. Figure 5 a displays the 

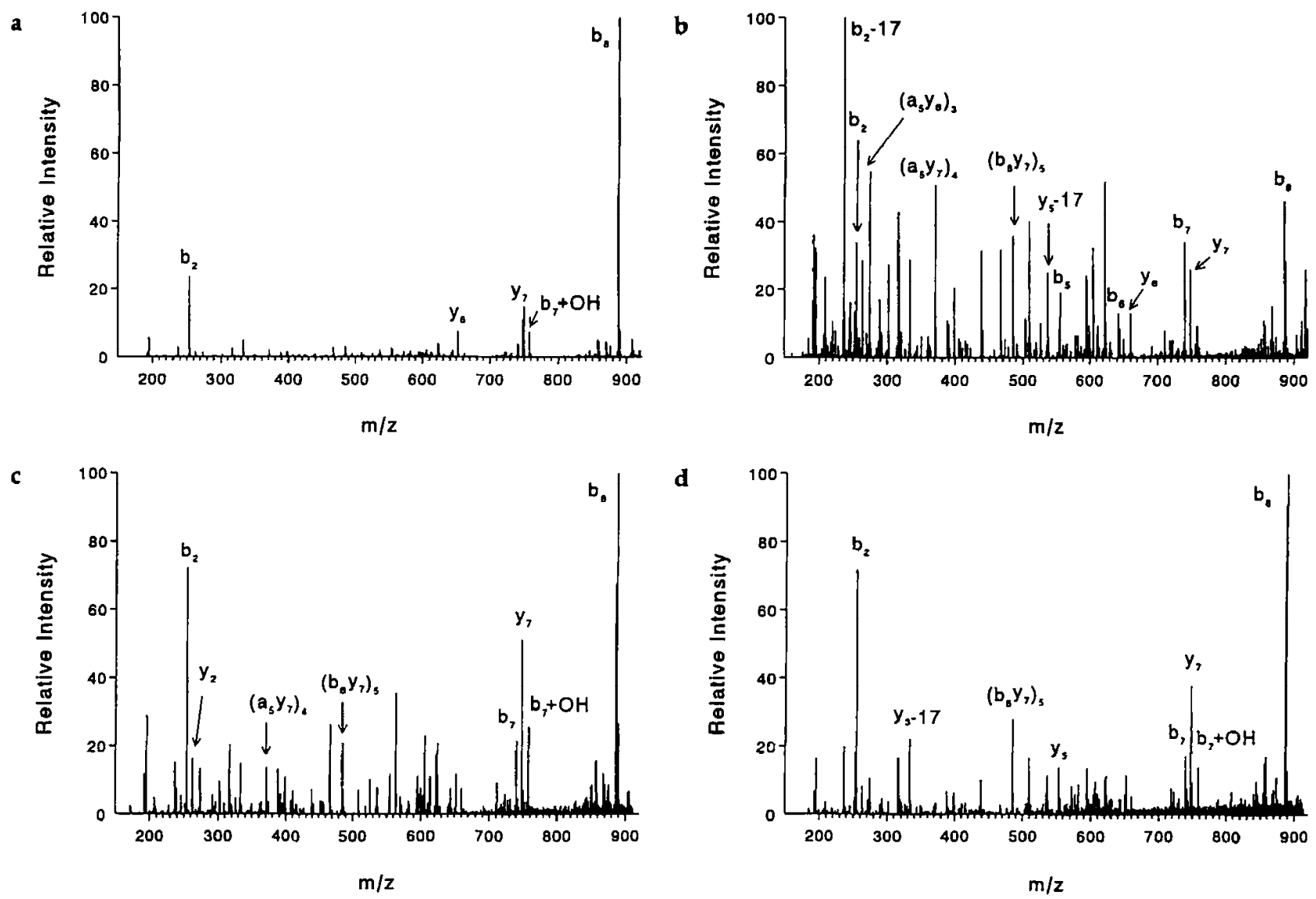

Figure 5. MS/MS of des-Arg'-bradykinin in the presence of helium only at $q_{z}=0.161$ (a). MS $/$ MS of des-Arg ${ }^{9}$-bradykinin at $q_{z}=0.161$ with a $500-\mu$ s pulse of krypton followed by a 15-ms delay (b), a 60 -ms delay (c), and a 150-ms delay (d) before resonant excitation.

MS/MS spectrum of des- Arg $^{9}$-bradykinin at a resonant excitation voltage of $600 \mathrm{mV}$ with only helium present. The MS/MS spectrum that results after a $500-\mu \mathrm{s}$ pulse of krypton at the same resonant excitation voltage can be seen in Figure 5b. Clearly, there are more product ions present in the latter spectrum with the potential for more useful information. Of particular interest is the presence of a number of low mass-tocharge ratio ions. Another appealing feature of the pulsed introduction of heavy gases is the ability to perform CID at $q_{z}$ values as low as 0.050 . This attribute allows the mass-to-charge ratio range over which product ions can be detected to be increased. Doroshenko and Cotter [19] demonstrated that this could be accomplished while still obtaining full sequence information for peptides. It should be noted that when CID is performed at such low $q_{z}$ values in the presence of helium alone, little to no dissociation can be seen; typically the ions are resonantly ejected [4].

Immonium ions in the MS/MS spectra of peptides are usually considered to be characteristic of high energy CID spectra. As was previously reported and as we have seen, immonium ions can be seen in relatively great abundance in the MS/MS spectra of peptides when the heavy gases are introduced via the pulsed method [19]. In fact for some five and six residue peptides, conditions can be chosen so that the immonium ions dominate the MS/MS spectra. The relatively high abundance of immonium ions raises the question, how are these ions formed? Are the collision conditions energetic enough to lead to direct formation of these ions? Alternatively, are these ions being formed after consecutive reactions, in which an intermediate product ion is first formed and then dissociates before acquisition of the spectrum? The absence of peaks corresponding to side chain cleavages (especially in the MS/MS spectrum of des-Arg ${ }^{9}$-bradykinin) seems to suggest that the collision conditions are not all that energetic and the presence of the lower mass-to-charge ratio ions are the result of consecutive fragmentations.

Ion motion simulations performed by using ITSIM provide insight into the kinetic energies of ions in the quadrupole ion trap under various conditions. Simulations show that when an ion is no longer in resonance, there is a finite time in which its kinetic energy is damped, and it is cooled to the center of the quadrupole ion trap. The time that it takes for an ion's kinetic energy to be effectively damped to ambient energies depends on the ion's kinetic energy during resonant excitation, the pressure of the background gas, and the $q_{z}$ value at which the ion is stored. It was observed 
that for a range of $q_{z}$ values, background gas pressures, and ion kinetic energies, the time for an ion's kinetic energy to be damped to ambient values was between 1 and $5 \mathrm{~ms}$. So, it is possible that when a product ion is formed and thus is no longer in resonance with the applied signal, the product ion can still undergo energetic collisions before its kinetic energy is effectively removed. If some of these energetic collisions are with a heavy gas, enough internal energy could be deposited to result in further dissociation. During our pulsed valve experiments a heavy gas pressure of about 1 mtorr has been estimated. Under such conditions, in a 3-ms time period, an ion would undergo about 40 collisions with xenon atoms, although with continuously decreasing $E_{\text {com }}$ because $E_{\text {lab }}$ is decreasing. However, this number of collisions may be sufficient to impart enough internal energy into the product ion to dissociate it further.

The pulsed mode of operation also affords some other advantages beyond increased energy deposition. By varying the delay time between when the heavy gas is pulsed in and when the resonant excitation signal is applied, the appearance of the resulting MS/MS spectra can be changed. Figure 5 shows the MS/MS spectra of des- $\mathrm{Arg}^{9}$-bradykinin with a resonant excitation voltage of $600 \mathrm{mV}$ as the delay time is increased from (c) $60 \mathrm{~ms}$ to (d) $150 \mathrm{~ms}$ after pulsing in krypton. These two spectra can be compared to the spectrum in Figure $5 b$ in which the delay time is 15 ms. By changing this delay time, the number density of the heavy gas under which resonant excitation is being performed is changed. In going from a $15-\mathrm{ms}$ delay to a $150-\mathrm{ms}$ delay before resonant excitation, the krypton has been pumped away to a greater extent. As a result, during CID the protonated species of desArg ${ }^{9}$-bradykinin is undergoing a decreased number of collisions with krypton and less energy is deposited into the ion. The ability to change the appearance of the MS/MS spectra can be useful in many ways. For example, the spectrum in Figure $5 b$ contains a complex variety of product ions which makes the interpretation of the spectrum very difficult. By increasing the delay time after the pulse of gas and before resonant excitation, the MS/MS spectrum can be "tuned" to give the appropriate amount of structural information (e.g., Figure $5 \mathrm{c}$ ). Obtaining spectra after different delay times may also be helpful in elucidating unknown product ions. The intensity of a peak could be monitored as the conditions were changed from a high density of heavy gas (high energy) to a low density of heavy gas (low energy), and this data may be useful in determining to what type of product ion the peak corresponds. Table 2 shows a breakdown of the distinct types of ions that can be confidently assigned (i.e., have relative intensities $>10 \%$ ) in the MS/MS spectra of des-Arg ${ }^{9}$ bradykinin at different delay times. At shorter delay times the number of internal fragment (i) ions is greater than other types of ions. The internal fragments are expected to be favored as the energy in the system is
Table 2. Number of peaks corresponding to various product ion types in tandem mass spectra of des-Arg ${ }^{9}$-bradykinin at different delay times after pulse of krypton

\begin{tabular}{lccc}
\hline Delay time & i-Type ions & b-Type ions & y-Type ions \\
\hline \hline $15 \mathrm{~ms}$ & 8 & 5 & 3 \\
$60 \mathrm{~ms}$ & 6 & 4 & 3 \\
$150 \mathrm{~ms}$ & 4 & 3 & 3 \\
Helium only & 0 & 2 & 2 \\
\hline
\end{tabular}

increased. At these shorter delay times the number density of the krypton is greater so the parent ions undergo more collisions with this heavy gas, and a greater amount of energy can be deposited into the ions. The ability to vary the delay could also be useful in chromatographic experiments. MS/MS on an analyte eluting from a column could be performed multiple times, each with a different delay time between the introduction of the heavy gas and the resonant excitation signal. This experiment would allow a number of MS/MS spectra under different energetic conditions to be obtained without having to change the resonant excitation voltage and deal with the change in the optimum resonant frequency.

Another advantage that pulsing in the heavy gases provides is a greater resonant excitation frequency range over which ions can be effectively dissociated at a given resonant excitation voltage. Figure 6 displays the intensity of the $[\mathrm{M}+\mathrm{H}]^{+}$species of leucine enkephalin at a resonance excitation voltage of $500 \mathrm{mV}$ and a $q_{z}=0.163$ as a function of the resonant excitation frequency with (a) helium and (b) while pulsing in argon with a 15-ms delay before resonant excitation. It can be clearly seen that the frequency range over which the protonated species is fully dissociated is much greater when argon is present versus helium alone. The vertical line in the plots represents the calculated theoretical secular frequency of the ion. This effect is even more pronounced when xenon is introduced via the pulsed valve. The widths of these resonance absorption profiles at $50 \%$ attenuation are 400 , 900 , and $1870 \mathrm{~Hz}$ for helium, argon, and xenon, respectively. If the resonant excitation voltage is changed, the width and the position of these profiles changes in the same manner as was previously reported [9]. Changing the number of ions present shifts the resonant absorption curves only slightly and changing the $q_{z}$ value at which the ions are resonantly excited does not significantly affect the widths of the profiles, but the position with respect to the theoretical frequency shifts noticeably.

The notable increase in the frequency range over which the parent ions can be dissociated is expected when the heavy gases are present. A collision between an ion and a heavy gas, such as argon, will deposit more energy per collision than will a corresponding collision with helium. Therefore, to achieve a given $E_{\text {com }}$, the kinetic energy of ions can be lower with heavier gases than with helium. The further the ap- 

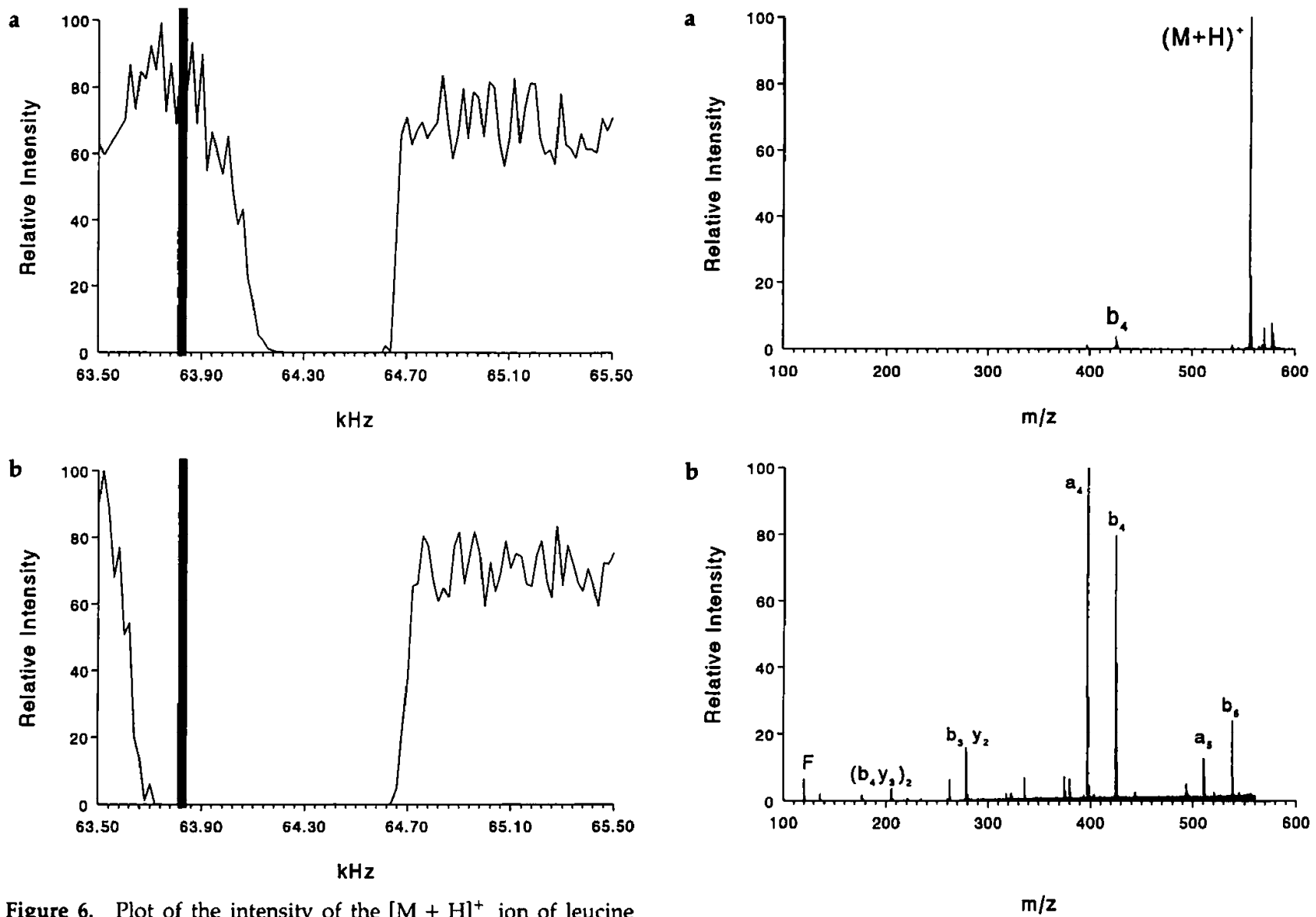

Figure 6. Plot of the intensity of the $[\mathrm{M}+\mathrm{H}]^{+}$ion of leucine enkephalin versus the frequency of the applied resonant excitation signal in the presence of helium (a) and a 500- $\mu$ s pulse of argon with a 15-ms delay before the application of the resonant excitation signal (b). The vertical solid lines indicate the ion's theoretically predicted secular frequency.

plied resonant excitation frequency is from the exact secular frequency of an ion, the less an ion's kinetic energy is increased. So, if an ion's kinetic energy is only slightly enhanced (i.e., excitation frequency shifted away from the exact ion secular frequency), but is in the presence of a heavy gas, similar amounts of internal energy can be deposited during a collision with the heavy gas as a collision with helium at higher kinetic energies (i.e., excitation frequency closer to exact ion secular frequency). This effect explains the increase in the excitation frequency range over which parent ions can be dissociated when heavy gases are present.

Because the resonant excitation frequency range over which dissociation occurs increases drastically with the introduction of heavy gases, the need to empirically optimize the applied resonant frequency is not as great. Figure 7 displays the MS/MS spectra of leucine enkephalin at a $q_{z}=0.163$, a resonant excitation voltage of $500 \mathrm{mV}$, and at the theoretical resonant frequency $(63,828 \mathrm{~Hz})$ with (a) helium and (b) a $500-\mu \mathrm{s}$ pulse of argon with a 15-ms delay before application of the resonant excitation signal. In the presence of helium alone (Figure 7a) there is virtually no dissocia-

Figure 7. MS/MS of leucine enkephalin after the application of the theoretically predicted resonant excitation frequency in the presence of helium (a) and a 500- $\mu$ s pulse of argon with a 15-ms delay before the application of resonant excitation signal (b).

tion, but in the presence of argon (Figure $7 \mathrm{~b}$ ) the amount of dissociation is more extensive.

\section{Conclusions}

The addition of a heavy gas during CID allows greater conversion of kinetic energy to internal energy in an ion. Thus, higher internal energies can be achieved during resonant excitation of the parent ion. Additionally, significant increases in internal energy of product ions can occur during the collisional damping of these ions after they are formed, leading to further dissociation of product ions. The increased internal energy deposition into the parent and product ions allows higher energy dissociation pathways to be accessed, which can be particularly important in obtaining structural information from large molecules such as peptides.

The pulsed introduction of heavy gases overcomes some deleterious effects that static pressures of these gases have on the performance of the quadrupole ion trap. Some of the limitations of performing CID by single frequency resonant excitation can be overcome with the pulsed introduction of heavy gases also. The 
amount of internal energy can be increased and good MS/MS efficiency can be obtained for an ion when applying the theoretical resonant excitation frequency. This result could be very useful when trying to perform CID by single frequency resonant excitation on a signal that is very short lived, because empirical optimization of the applied signal otherwise may be too time consuming. Also by varying the delay time between the pulse of the heavy gas and the application of the resonant excitation signal, MS/MS spectra can be tuned to provide the appropriate amount of structural information. One disadvantage with pulsed introduction of the heavy gases is the increased time required to perform the experiments. The length of the experiments described here was the result of extended times to ensure the complete evacuation of the heavy gases before acquisition. It is expected that the time for the removal of the heavy gas can be reduced without detrimental effects on the resulting spectra. The advantages obtained by pulsed introduction of heavy gases should extend the analytical utility of the quadrupole ion trap in the structural studies of biomolecules.

\section{Acknowledgment}

This work was supported by NIH GM49852.

\section{References}

1. Louris, J. N.; Cooks, R. G.; Syka, J. E. P.; Kelley, P. E.; Stafford, G. C.; Todd, J. F. J. Anal. Chem. 1987, 59, 1677.

2. March, R. E.; Hughes, R. J. Quadrupole Storage Mass Spectrometry; Wiley: New York, 1989.

3. Cooks, R. G.; Glish, G. L.; McLuckey, S. A.; Kaiser, R. E. Chem. Eng. News 1991, 69, 26.
4. Charles, M. J.; McL.uckey, S. A.; Glish, G. L. J. Am. Soc. Mass Spectrom. 1994, 5, 1031.

5. Todd, J. F. J.; Waldren, R. M.; Mather, R. E. Int. I. Mass Spectrom. Ion Phys. 1980, 34, 325.

6. Todd, J. F. J.; Waldren, R. M.; Freer, D. A.; Turner, R. B. Int. J. Mass Spectrom. Ion Phys. 1980, 35, 107.

7. Franzen, J. Int. I. Mass Spectrom. Ion Processes 1991, 106, 63.

8. Vedel, F.; Vedel, M. Phys. Rev. A 1990, 41, 2348.

9. Williams, J. D.; Cox, K. A.; Cooks, R. G.; McLuckey, S. A.; Hart, K. J.; Goeringer, D. E. Anal. Chem. 1994, 66, 725.

10. Lammert, S. A.; Cooks, R. G. J. Am. Soc. Mass Spectrom. 1991, $2,487$.

11. Paradisi, C.; Todd, J. F. J.; Vettori, U. Org. Mass Spectrom. 1992, 27, 251.

12. Paradisi, C.; Todd, J. F. J.; Vettori, U., Org. Mass Spectrom. 1992, 27, 1210.

13. Curcuruto, O.; Fontana, S.; Traldi, P.; Celon, E. Rapid Commun. Mass Spectrom. 1992, 6, 322.

14. Creaser, C. S; O'Neill, K. E. Org. Mass Spectrom. 1993, 28 , 564.

15. March, R. E.; Weir, M. R.; Londry, F. A.; Catinella, S.; Traldi, P.; Stone, J. A.; Jacobs, W. B. Can. J. Chem. 1994, 72, 966.

16. Gronowska, J.; Paradisi, C.; Traldi, P.; Vettori, U. Rapid Commun. Mass Spectrom. 1990, 4, 307.

17. Nourse, B. D.; Cox, K. A.; Morand, K. L.; Cooks, R. G. J. Am. Chem. Soc. 1992, 114, 2010.

18. Morand, K. L.; Cox, K. A.; Cooks, R. G. Rapid Commun. Mass Spectrom. 1992, 6, 520 .

19. Doroshenko, V. M.; Cotter, R. J. Anal. Chem. 1996, 68, 463.

20. Van Berkel, G. J.; Glish, G. L.; McLuckey, S. A. Anal. Chem. 1990, 62, 1284.

21. Reiser, H.-P.; Julian, R. K., Jr.; Cooks, R. G. Int. I. Mass Spectrom. Ion Processes 1992, 121, 49.

22. Alexander, A. J.; Boyd, R. K. Int. J. Mass Spectrom. Ion Processes 1989, 90, 211.

23. Thibault, P.; Alexander, A. J.; Boyd, R. K.; Tomer, K. B. J. Am. Soc. Mass Spectrom. 1993, 4, 845.

24. McLuckey, S. A. J. Am. Soc. Mass Spectrom. 1992, 3, 599. 